\title{
Inelastic scattering of light by a cold trapped atom: Effects of the quantum center-of-mass motion
}

\author{
Marc Bienert \\ Centro de Ciencias Físicas UNAM, Campus Morelos UNAM, 62251 Cuernavaca, Morelos, Mexico \\ Wolfgang Merkel \\ Abteilung für Quantenphysik, Universität Ulm, Albert-Einstein-Allee 11, 89069 Ulm, Germany \\ Giovanna Morigi \\ Grup d'Optica, Departament de Fisica, Universitat Autonoma de Barcelona, 08193 Bellaterra, Spain
}

(Received 16 November 2005; published 2 March 2006)

\begin{abstract}
The light scattered by a cold trapped ion, which is in the stationary state of laser cooling, presents features due to the mechanical effects of atom-photon interactions. These features appear as additional peaks (sidebands) in the spectrum of resonance fluorescence. Among these sidebands the literature has discussed the Stokes and anti-Stokes components: namely, the sidebands of the elastic peak. We show that the motion also gives rise to sidebands of the inelastic peaks. These are not always visible, but, as we show, can be measured in parameter regimes which are experimentally accessible.

DOI: 10.1103/PhysRevA.73.033402

PACS number(s): 32.80.Pj, 42.50.Vk
\end{abstract}

\section{INTRODUCTION}

The mechanical effects of photon-atom interactions are at the basis of several techniques of manipulation of the quantum state of atoms [1]. Their understanding is therefore relevant for implementations of coherent control. Features due to the center-of-mass motion have been measured in the spectrum of resonance fluorescence of trapped atoms [2] and ions [3-7]. These measurements allowed one to gain insight into the state of the laser-cooled atoms and were at the basis of the implementation of cooling schemes on the atomic motion based on feedback control [8].

The spectrum of resonance fluorescence of a laser-cooled atom is obtained from the spectral analysis of the intensity of the scattered light. By means of these scattering processes the atomic center-of-mass motion is cooled by transferring center-of-mass energy into the photonic reservoir. Hence, the scattered photons carry information about the dynamics that the atom undergoes, which can be partly reconstructed in the power spectrum. Most studies done so far on the resonance fluorescence of trapped ions focused on the elastically scattered radiation by a confined atomic dipole and on its Stokes and anti-Stokes sidebands, thereby finding good agreement between theory and experiment [2-11]. On the other hand, the effects of the center-of-mass motion on the inelastic part of the spectrum of resonance fluorescence are largely unexplored. Features of mechanical effects in this radiation, which has no classical analog, have been studied in [12] for the case of a trapped atom, undergoing laser cooling by driving two coupled dipole transitions in the regime where fluorescence is solely due to the mechanical effects of light. In [12] it was found that the quantum motion gives rise to sidebands of the inelastic spectrum, which can be mapped to Raman processes between the dressed states of the bare three-level transition.

In this work we investigate the spectrum of resonance fluorescence of a trapped atom whose dipole transition is driven by the cooling laser, and we address in particular the issue whether and how features due to the mechanical effects of light can be identified and measured in the inelastic part of the spectrum. Indeed, we show that the quantum motion gives rise to sidebands of the peaks of the inelastic spectrum, which have different properties compared to the sidebands of the elastic component. These sidebands are not always visible; however, they can be measured in experimentally accessible parameter regimes, like, for instance, in [13].

The investigation in this work complements the theoretical study of [10], which focused on the Stokes and antiStokes components of the spectrum of resonance fluorescence of a driven dipole. Like in [10], we consider the situations when the cooling laser is (i) a running wave and (ii) a standing wave. The spectrum is calculated by using the spectral decomposition of the atomic master equation [14-16]

This work is organized as follows. In Sec. II we present and discuss the spectra of resonance fluorescence. In Sec. III the theoretical model at the basis of the derivation is reported. The conclusions are drawn in Sec. IV. The appendixes report further details of the derivations of Sec. III.

\section{SPECTRUM OF RESONANCE FLUORESCENCE}

Let us consider the radiation scattered by a trapped atom in the latest stages of laser cooling and detected at a narrowband detector, in the setup sketched in Fig. 1. The laser drives the atomic dipole transition, and the atomic motion is laser cooled to a stationary state, which is determined by the statistical equilibrium between scattering processes changing the motional excitations. The scattered photons originate from these processes, and the correlation functions and corresponding spectra contain some features of these dynamics. The spectrum of the intensity of the scattered radiation at the detector is [9] 


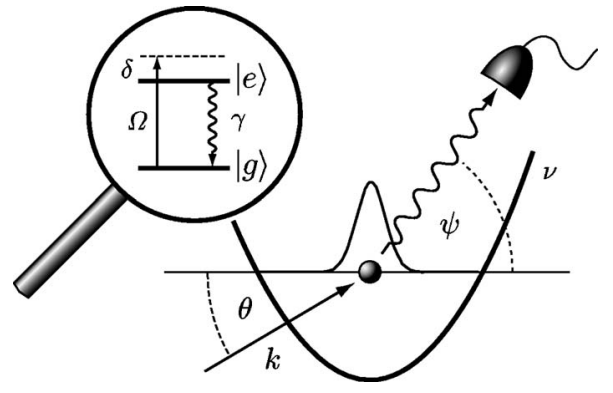

FIG. 1. An atom is trapped in a harmonic potential of frequency $\nu$, and its dipole transition is driven by a laser field with wave vector $k$ and propagating at angle $\theta$ with the axis of motion. The laser cools the atomic motion, and a narrow-band detector records the spectrum of the intensity of the light which is scattered at angle $\psi$. The Gaussian function denotes the center-of-mass wave packet, whose finite size affects the properties of the scattered light. The inset shows the relevant internal atomic structure, composed of the ground and excited states $|g\rangle$ and $|e\rangle$, which decays at rate $\gamma$ into $|g\rangle$. Here, $\Omega$ and $\delta$ denote the laser Rabi frequency and detuning, respectively.

$$
S(\omega)=\chi \operatorname{Re} \int_{0}^{\infty} \mathrm{d} \tau \mathrm{e}^{-\mathrm{i} \omega \tau}\left\langle E^{(+)}(t+\tau) E^{(-)}(t)\right\rangle,
$$

where $\chi$ is a constant, $E^{(+)}(t)\left[E^{(-)}(t)\right]$ are the negative [positive] frequency part of the electric field at the detector at time $t$, and $\langle\cdot\rangle$ describes the average over the atom and electromagnetic field degrees of freedom. For suitably chosen detection angles the field at the detector is the field scattered by the source, and it is thus determined by the state of the atom.

Let us now make some preliminary considerations and discuss in particular the form of the spectrum when the features due to the mechanical effects are negligible. In this regime, the spectrum of resonance fluorescence of a dipole transition is constituted by two contributions: the elastic component-namely, a narrow peak at the frequency $\omega_{L}$ of the laser-corresponding to Rayleigh scattering and the inelastic component, which originates from quantum fluctuations of the atomic dipole. This part exhibits the well-known peaks of the Mollow triplet which is found when the transition is driven at saturation $[17,18]$.

The mechanical effects of light clearly modify the form of this spectrum. The atomic motion, confined by a harmonic potential of frequency $\nu$, couples to the scattered light. This gives rise to sidebands of the elastic peak, whose origin can be intuitively understood on the basis of a classical model for the atomic motion [19]. In the regime where the atomic wave packet is localized over the wavelength of light, the mechanical coupling is small and scales with the Lamb-Dicke parameter $\eta \ll 1$. In this regime only the Stokes and antiStokes sidebands are visible. At low temperatures the form of these sidebands depends critically on the detection angle. This dependence is due to interference between quantum paths of excitations coupling internal and external degrees of freedom [10].

The inelastic component of the spectrum of resonance fluorescence has no classical analog. The mechanical effects on this part mainly consist of the appearance of sidebands of the inelastic peaks. As we discuss below, these sidebands share some properties with but also substantial differences from the sidebands of the elastic peak.

\section{Results}

In this section we report and discuss the theoretical results, whose derivation is reported in Sec. III. Figure 2 displays the spectra of the light scattered by the dipole transition of an ion which is sideband cooled [19,20]. The spectra have been evaluated for different parameter regimes. Figures 2(a) and 2(b) correspond to the spectrum at two different detection angles in the situation in which the linewidth of the dipole transition $\gamma=\nu / 3$. Here, the mechanical effects of atom-photon interactions are clearly evident in the sidebands of the elastic peak, the two narrow signals at frequency $\omega_{L} \pm \nu$. Their width scales with the recoil frequency and corresponds with the cooling rate $[9,10,12]$. These resonances are magnified in the inset, where one can see that their centers are slightly shifted from the values $\omega_{L} \pm \nu$. This shift is due to the mechanical coupling $[10,12]$. The corresponding curves are a superposition of Lorentz and Fano-like profiles, whereby one functional dependence can dominate over the other depending on the angle of observation. The asymmetry of these peaks is due to interference effects, which originate from the quantum nature of the ion center-of-mass motion and whose magnitude depends on the detection angle [10]. Manifestations of the mechanical effects in the inelastic spectrum are small but yet visible in Fig. 2(b), causing the appearance of an additional resonance on the left part of the curve (at $\omega=\omega_{L}-2.4 \nu$ ) and a broadening of the central inelastic peak. The width of the sidebands of the inelastic peaks is substantially the same as the one of the corresponding inelastic peak at zero order in the motion. Their height scales with the recoil frequency, and like the sidebands of the elastic peak, it is a nontrivial function of the average phononic excitation $\langle n\rangle$ and of the angle of detection. Their functional form is reported in Sec. III B.

Figures 2(c) and 2(d) display the spectra of fluorescence when the linewidth $\gamma=\nu / 10$. Here, the mechanical effects can be clearly identified in the inelastic spectrum, displaced by about $\pm \nu$ from the corresponding signal of the Mollow triplet. The sidebands of the central inelastic peak are centered at the same frequency as the Stokes and anti-Stokes signals, and give rise to a small broadening at their basis.

We remark that the height of the mechanical sidebandsfor elastic and inelastic scattering-critically depends on the detection angle, as is visible in the figures. Also for the inelastic spectrum this effect is caused by interference of the underlying atomic processes contributing to each spectral signature and depending on the direction of photon absorption and emission. Thus, the behavior also depends on whether the drive is a traveling wave, as in Fig. 2, or a standing wave. Figure 3 shows the spectrum of an ion where the trap center is in a point of the standing wave (away from the nodes and antinodes) for $\gamma=\nu / 10$ and at two different detection angles. Here, one can observe the dependence of the sidebands of the elastic and inelastic part on the detection 
a)

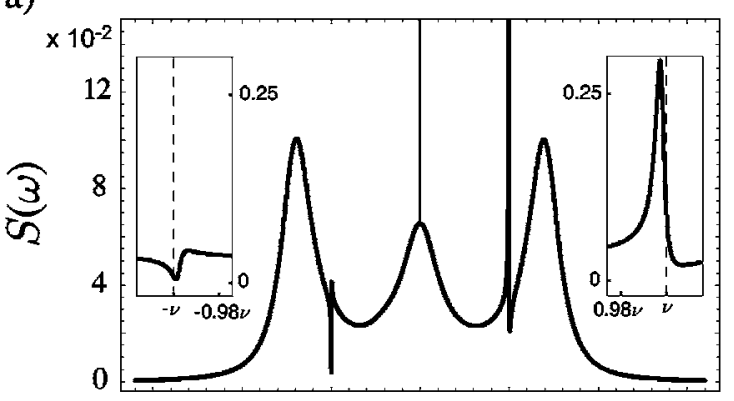

c)

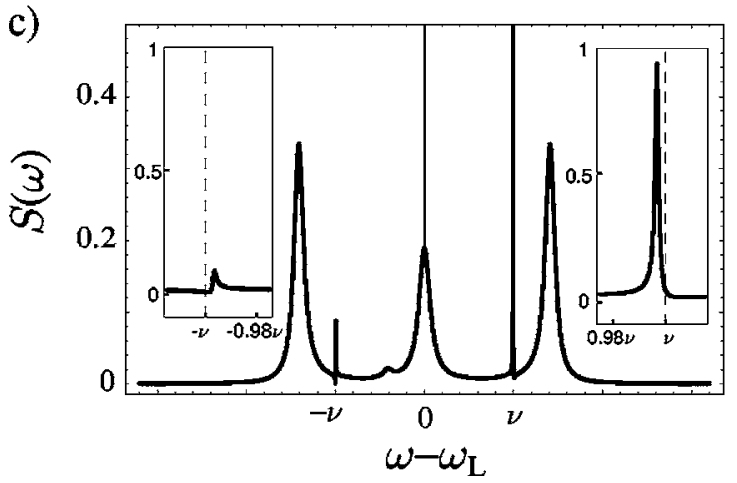

b)

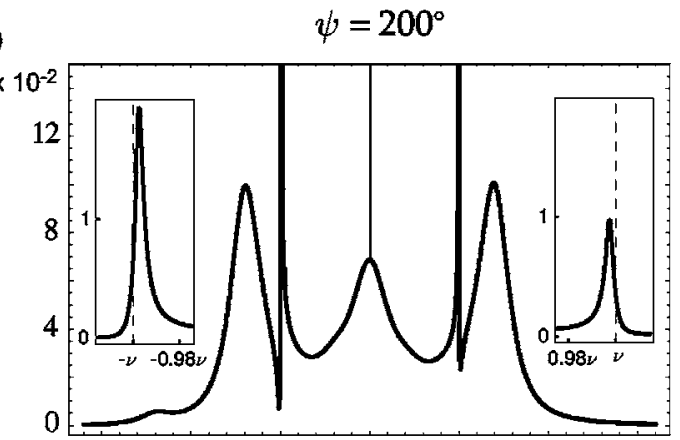

d)

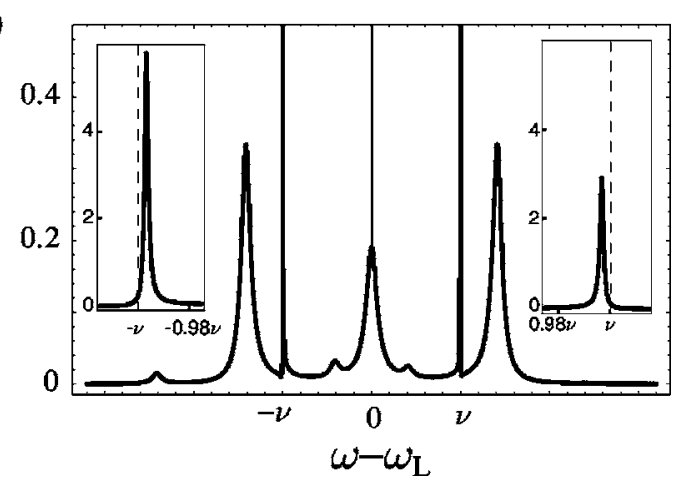

FIG. 2. Spectrum of the intensity (in arbitrary units) of the light scattered by the dipole transition of a trapped ion in the Lamb-Dicke regime. The ion is sideband cooled by a traveling-wave laser. The parameters are $\delta=-\nu, \Omega=\nu, \eta=0.1$, and (a), (b) $\gamma=0.33 \nu$, corresponding to average phononic occupation $\langle n\rangle=0.15$; (c), (d) $\gamma=0.1 \nu(\langle n\rangle=0.14)$. Spectra (a) and (c) are measured at the detection angle $\psi=40^{\circ}$, spectra (b) and (d) at $\psi=200^{\circ}$. The plots show the elastic peak, symbolized by the thin line at frequency $\omega=\omega_{L}$, the Mollow spectrum, and the motional sidebands of the Mollow peaks. The thin and outstanding peaks at about $\omega_{L} \pm \nu$ are the sidebands of the elastic peak. A magnification of these contributions is shown in the insets.

angle. Figure 4 displays the spectra of resonance fluorescence at various points of the standing wave. For $\varphi=\pi / 4$, Fig. 4(a), the spectrum is governed by the outstanding sidebands of the elastic peak, overtopping the Mollow triplet. At $\varphi=3 \pi / 8$, Fig. 4(b), the trap is closer to the node of the standing wave. Here, the resonances of the Mollow triplet get closer. In this case, the height of the sidebands of the elastic peak decreases and the sidebands of inelastically scattered light are visible. A peculiar case is found when the atom is placed at the node, Fig. 4(c), at $\varphi=\pi / 2$. Here, the only spectral signals are the motional sidebands. These sidebands are now perfect Lorentzian curves of identical shape independent of the detector position, as already pointed out in [10]. What is novel is that all other contributions of the spectrum, elastic and inelastic, disappear due to the vanishing of the driving field at the center of the trap. In particular, even the motional sidebands of the inelastic spectrum disappear. The appearance of the sidebands of the elastic peak is due to the effects of the gradient of the field intensity over the atomic wave packet at the node. The suppression of inelastic scattering processes, included the ones that change the motional excitation, can be understood by considering that there are no quantum fluctuations of the atomic dipole at the node of a standing wave.

These behaviors are discussed in detail in the following theoretical treatment. It should be remarked that the sidebands of the inelastic component become visible when the transition, at zero order in the mechanical effects, is driven at saturation. Below saturation the mechanical effects in the inelastic spectrum manifest in small sidebands of the Lorentz curve centered at the laser frequency. These sidebands are at the same frequencies as the ones of the Stokes and antiStokes components of the elastic peak, and are covered by these signals.

\section{EVALUATION OF THE SPECTRUM OF RESONANCE FLUORESCENCE}

We consider an atom of mass $M$, whose center-of-mass motion is confined by a harmonic potential of frequency $\nu$. We assume that the relevant motion is along one dimension, the $x$ axis, while the radial motion is frozen out. A laser, of frequency $\omega_{L}$ and wave vector $k$, couples quasiresonantly with the atomic dipole transition between the ground and excited states $|g\rangle$ and $|e\rangle$ at frequency $\omega_{0}$ and linewidth $\gamma$. The motion is in the Lamb-Dicke regime; namely, the size of the center-of-mass wave packet is assumed to be much smaller than the laser wavelength and the dipole linewidth $\gamma<\nu$. In this regime the laser can be set at frequency $\omega_{L}$ $=\omega_{0}-\nu$, thereby sideband-cooling the motion $[19,20]$. The atomic motion is in the stationary state of sideband cooling and scatters the laser light.

Let a detector measure the radiation scattered at the angle $\psi$ with the atom motional axis. In the far field the scattered field is proportional to the retarded value of the atomic di- 
a)

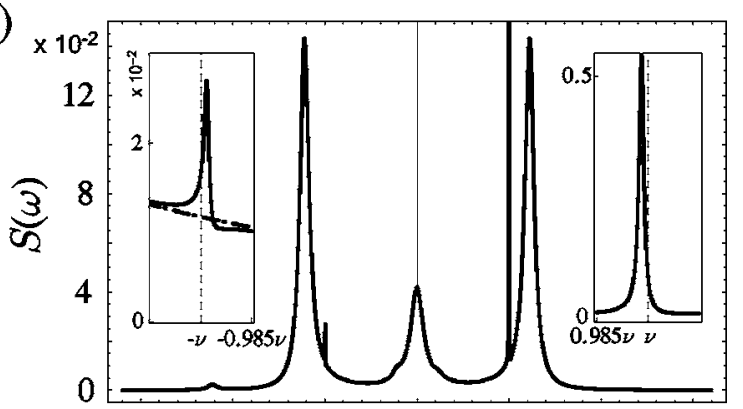

b)

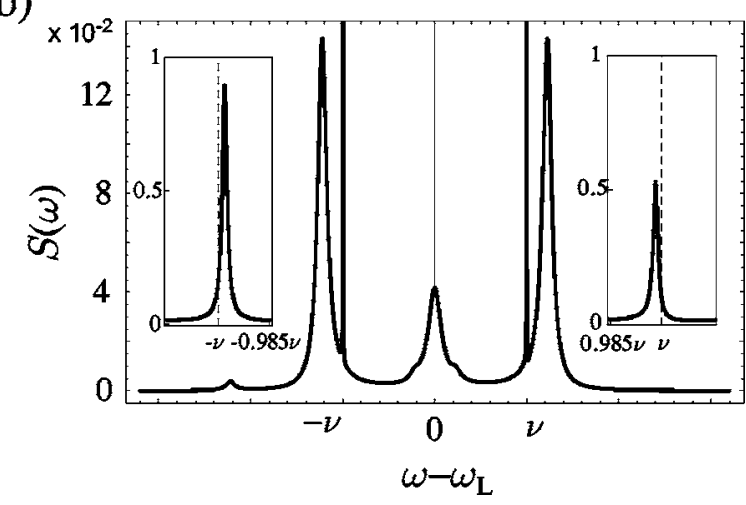

FIG. 3. Spectrum of the intensity (in arbitrary units) of the light scattered by the dipole transition of a trapped ion in the LambDicke regime. The ion is sideband cooled by a standing-wave laser. The parameters are $\delta=-\nu, \Omega=\nu, \gamma=0.1 \nu, \eta=0.1$, and detector angle (a) $\psi=40^{\circ}$ and (b) $\psi=200^{\circ}$. The standing wave is described by the function $\cos (k x+\varphi)$ where $\varphi=\pi / 4$ is the value at the trap center. The mean phononic excitation is $\langle n\rangle=0.04$. The insets display the sidebands of the elastic peak; the dash-dotted lines show the inelastic spectrum contribution.

pole at the corresponding position $x$. The spectrum at frequency $\omega$ is given by [21]

$$
S(\omega)=\tilde{\chi}(\psi) \operatorname{Re} \int_{0}^{\infty} \mathrm{d} \tau \mathrm{e}^{-\mathrm{i}\left(\omega-\omega_{L}\right) \tau}\left\langle D^{\dagger}(x, \tau) D(x, 0)\right\rangle_{\mathrm{st}},
$$

where $\widetilde{\chi}(\psi)$ is a function of the emission angle and $D(x, t)$ is the dipole-lowering operator in the reference frame rotating with laser frequency $\omega_{L}$ at position $x$ of the atomic center of mass. The correlation function in Eq. (2) is formally evaluated by means of the quantum regression theorem, according to which $D(x, t)=D(x) \mathrm{e}^{\mathcal{L} t}$ with $\mathcal{L}$ the Liouvillian of the master equation for the atomic density matrix $\varrho$,

$$
\partial \varrho / \partial t=\mathcal{L} \varrho
$$

The average $\langle\cdot\rangle_{\mathrm{st}}$ is taken over the atomic density matrix $\varrho_{\mathrm{st}}$ at steady state, the solution of the equation $\mathcal{L} \varrho_{\text {st }}=0$.

The explicit form of $S(\omega)$, Eq. (2), is found by applying the spectral decomposition of the Liouville operator $\mathcal{L}$ according to the secular equations

$$
\mathcal{L} \varrho^{\lambda}=\lambda \varrho^{\lambda},
$$
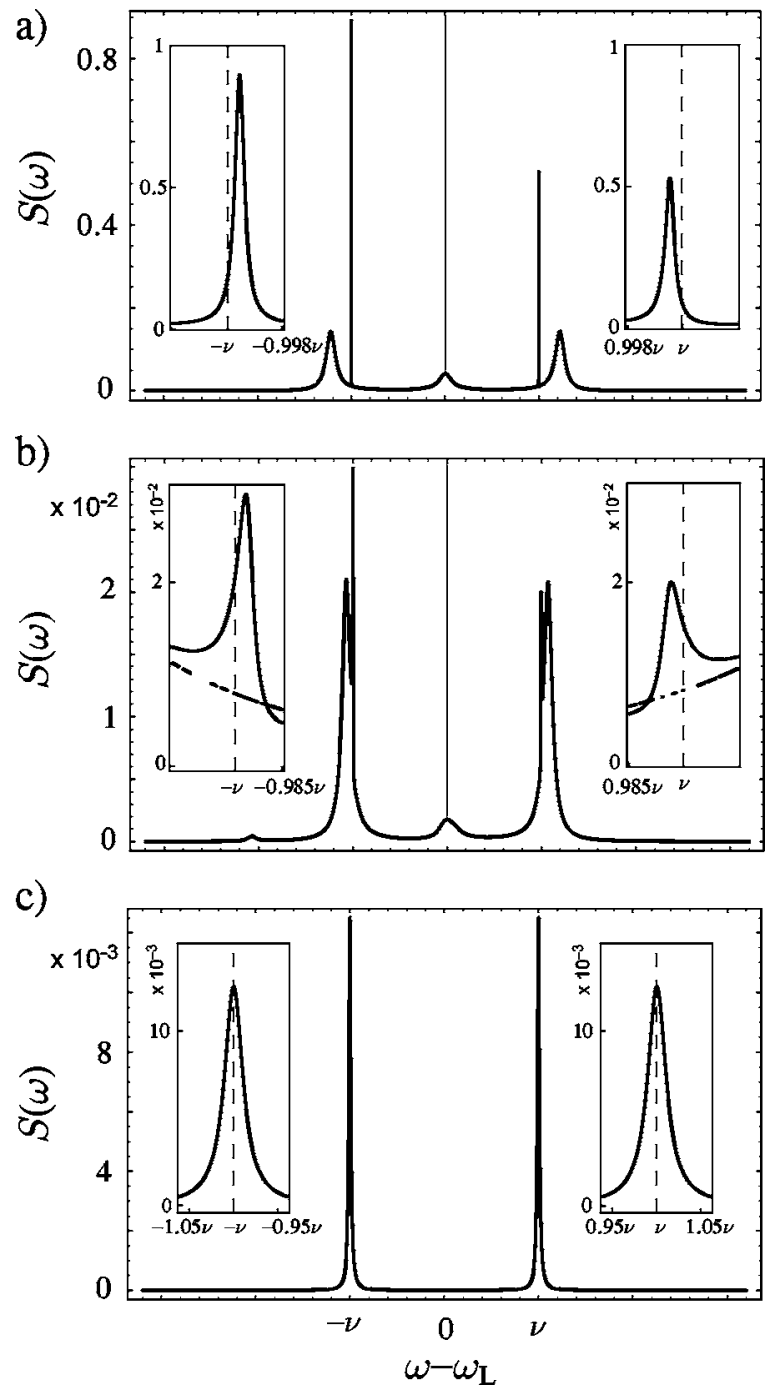

FIG. 4. Same as Fig. 3 for different positions in the standing wave. Here, (a) $\varphi=\pi / 4(\langle n\rangle=0.04)$, (b) $\varphi=3 \pi / 8(\langle n\rangle=0.002)$, and (c) $\varphi=\pi / 2 \quad(\langle n\rangle=0.0006)$. The parameters are $\delta=-\nu, \Omega=\nu, \gamma$ $=0.1 \nu, \eta=0.05$, and $\psi=200^{\circ}$. The insets display the sidebands of the elastic peak; the dash-dotted lines correspond to the inelastic spectrum contribution.

$$
\check{\varrho}^{\lambda} \mathcal{L}=\lambda \check{\varrho}^{\lambda},
$$

with eigenvalues $\lambda$ and right and left eigenelements $\varrho^{\lambda}$ and $\check{\varrho}^{\lambda}$, respectively $[14,15]$. The orthogonality and completeness of the eigenelements are defined with respect to the trace, such that $\operatorname{Tr}\left\{\check{\varrho}^{\lambda^{\prime}} \varrho^{\lambda}\right\}=\delta^{\lambda^{\prime}, \lambda}$. We define the projectors onto the eigenspace corresponding to the eigenvalue $\lambda$ as $\mathcal{P}^{\lambda}=\varrho^{\lambda}$ $\otimes \check{\varrho}^{\lambda}$ such that their action on an operator $X$ is defined as $\mathcal{P}^{\lambda} X=\varrho^{\lambda} \operatorname{Tr}\left\{\check{\varrho}^{\lambda} X\right\}$, and they thus fulfill $\mathcal{L} \mathcal{P}^{\lambda}=\mathcal{P}^{\lambda} \mathcal{L}=\lambda \mathcal{P}^{\lambda}$. By applying this formalism, we rewrite Eq. (2) as

$$
S(\omega)=\tilde{\chi}(\psi) \operatorname{Re} \sum_{\lambda} \frac{1}{\mathrm{i}\left(\omega-\omega_{L}\right)-\lambda} \operatorname{Tr}\left\{D^{\dagger}(x) \mathcal{P}^{\lambda} D(x) \varrho_{\mathrm{st}}\right\},
$$

where we have used the completeness relation of the eigenelements of $\mathcal{L}$. Although completeness of this kind of 
basis is generally not warranted, the spectral decomposition that we consider in the following is complete, and the spectrum of resonance fluorescence can be thus cast in the form of Eq. (4).

\section{A. Model}

We introduce now the basic elements determining the dynamics of the atom. The coupling with radiation is assumed to be in the Lamb-Dicke regime. This regime is characterized by the small value of the Lamb-Dicke parameter

$$
\eta=\sqrt{\frac{\hbar k^{2}}{2 M \nu}},
$$

which scales the coupling between internal and external atomic degrees of freedom due to photon scattering. We identify $\eta$ as the parameter of the perturbative expansion. According to this expansion, at second order the spectrum in Eq. (4) is decomposed into the terms

$$
S(\omega)=S_{0}(\omega)+S_{1}(\omega)+S_{2}(\omega)+O\left(\eta^{3}\right),
$$

where the subscripts label the corresponding order in the Lamb-Dicke expansion. In order to evaluate $S_{j}(\omega)$, we consider the operators $\mathcal{L}$ and $D$ at second order in the expansion in $\eta$. The dipole $D(x)=D_{0}+D_{1}+D_{2}+O\left(\eta^{2}\right)$, where the individual terms have the form

$$
\begin{gathered}
D_{0}=\sigma, \\
D_{1}=-\mathrm{i} k x \cos \psi \sigma, \\
D_{2}=-\frac{1}{2} k^{2} x^{2} \cos ^{2} \psi \sigma,
\end{gathered}
$$

with $\sigma=|g\rangle\langle e|$. The Liouville operator is decomposed into the terms $\mathcal{L}=\mathcal{L}_{0}+\mathcal{L}_{1}+\mathcal{L}_{2}$, which we now introduce in detail. At zero order internal and external degrees of freedom are decoupled: namely,

$$
\mathcal{L}_{0}=\mathcal{L}_{\mathrm{I}}+\mathcal{L}_{\mathrm{E}}
$$

where $\mathcal{L}_{\mathrm{E}}$ and $\mathcal{L}_{\mathrm{I}}$ act on the external and internal degrees of freedom, respectively. Here,

$$
\mathcal{L}_{\mathrm{E}} \varrho=\frac{1}{i \hbar}\left[H_{\mathrm{mec}}, \varrho\right],
$$

with

$$
H_{\mathrm{mec}}=\hbar \nu\left(a^{\dagger} a+\frac{1}{2}\right),
$$

where $a$ and $a^{\dagger}$ are the annihilation and creation operators of a quantum of energy $\hbar \nu$, respectively. We denote with $|n\rangle$ the eigenvectors of $H_{\text {mec }}$, fulfilling $H_{\text {mec }}|n\rangle=\hbar \nu(n+1 / 2)|n\rangle$ with $n=0,1,2, \ldots$. The term $\mathcal{L}_{\mathrm{I}}$ acts on the internal degrees of freedom, and it is defined as

$$
\mathcal{L}_{\mathrm{I}} \varrho=\frac{1}{i \hbar}\left[H_{0}+V_{0}, \varrho\right]+\mathcal{K} \varrho,
$$

where $H_{0}=\hbar \delta|g\rangle\langle g|$, with $\delta=\omega_{L}-\omega_{0}$, and

$$
\begin{gathered}
V_{0}=\frac{1}{2} \hbar \Omega \zeta(\varphi) \sigma^{\dagger}+\text { H.c. }, \\
\mathcal{K}_{0} \varrho=\frac{\gamma}{2}\left(2 \sigma \varrho \sigma^{\dagger}-\sigma^{\dagger} \sigma \varrho-\varrho \sigma^{\dagger} \sigma\right)
\end{gathered}
$$

are the interaction with radiation at zero order in the mechanical effects. Here, we have denoted by $\Omega \zeta(\varphi)$ the laser Rabi frequency, whereby $\zeta(\varphi)$ is a dimensionless function of the angle $\varphi$, which is $\zeta(\varphi)=\left.\exp (\mathrm{i} \varphi)\right|_{\varphi=0}$ for a traveling-wave drive, and is given by $\zeta(\varphi)=\cos \varphi$ for a standing-wave drive.

The first- and second-order Liouville operators give rise to the mechanical coupling between internal and external degrees of freedom,

$$
\begin{gathered}
\mathcal{L}_{1} \varrho=\frac{1}{\mathrm{i} \hbar}\left[x V_{1}, \varrho\right], \\
\mathcal{L}_{2} \varrho=\frac{1}{2 \mathrm{i} \hbar}\left[x^{2} V_{2}, \varrho\right]+\mathcal{K}_{2} \varrho,
\end{gathered}
$$

where

$$
\begin{gathered}
V_{1}=\frac{1}{2} k \cos \theta \Omega \zeta^{\prime}(\varphi) \sigma^{\dagger}+\text { H.c., } \\
V_{2}=\frac{1}{2} k^{2} \cos ^{2} \theta \Omega \zeta^{\prime \prime}(\varphi) \sigma^{\dagger}+\text { H.c. } \\
\mathcal{K}_{2} \rho=\beta \frac{\gamma}{2} k^{2} \sigma\left(2 x \rho x-x^{2} \rho-\rho x^{2}\right) \sigma^{\dagger} .
\end{gathered}
$$

Here, $\theta$ is the angle between the laser and trap axis, $\beta$ is a constant, which gives the average recoil due to spontaneous emission of a photon, and $\zeta^{\prime}(\varphi)$ and $\zeta^{\prime \prime}(\varphi)$ are the first and second derivatives of $\zeta(\varphi)$ with respect to $\varphi$.

The spectral decomposition of the Liouville operator is obtained using the eigenvalues and eigenelements of operator $\mathcal{L}_{0}$, of which we evaluate the corrections at second order in $\eta$. We denote the eigenvalues and eigenelements of $\mathcal{L}_{0}$ by $\lambda_{0}$ and $\varrho_{0}^{\lambda}, \check{\varrho}_{0}^{\lambda}$, respectively. Since internal and external degrees of freedom are decoupled at zero order in $\eta$, the eigenvalues of $\mathcal{L}_{0}$ are $\lambda_{0}=\lambda_{\mathrm{I}}+\lambda_{\mathrm{E}}$ and the eigenelements

$$
\varrho_{0}^{\lambda}=\rho^{\lambda_{\mathrm{I}}} \mu^{\lambda_{\mathrm{E}}},
$$

where $\mathcal{L}_{\mathrm{I}} \rho^{\lambda_{\mathrm{I}}}=\lambda_{\mathrm{I}} \rho^{\lambda_{\mathrm{I}}}$ and $\mathcal{L}_{\mathrm{E}} \mu^{\lambda_{\mathrm{E}}}=\lambda_{\mathrm{E}} \mu^{\lambda_{\mathrm{E}}}$. Correspondingly, the projector into the subspace at the eigenvalue $\lambda_{0}$ is

$$
\mathcal{P}_{0}^{\lambda}=\mathcal{P}^{\lambda} \mathcal{U}^{\lambda} \mathrm{E},
$$

whereby their action on the operator $X$ is defined as

$$
\begin{gathered}
\mathcal{P}^{\lambda} \mathrm{I} X=\rho^{\lambda} \operatorname{Tr}_{\mathrm{I}}\left\{\check{\rho}^{\lambda} \mathrm{I} X\right\}, \\
\mathcal{U}^{\lambda} \mathrm{E} X=\mu^{\lambda_{\mathrm{E}}} \operatorname{Tr}_{\mathrm{E}}\left\{\check{\mu}^{\lambda} \mathrm{E} X\right\},
\end{gathered}
$$

and $\operatorname{Tr}_{\mathrm{I}}\left(\operatorname{Tr}_{\mathrm{E}}\right)$ denotes the trace over the internal (external) degrees of freedom.

The spectrum of $\mathcal{L}_{\mathrm{I}}$ characterizes the dynamics of the two-level transition and the spectral properties of the radia- 
tion emitted by the bare atom [16]. The eigenvalues of $\mathcal{L}_{\mathrm{E}}$ take on the values $\lambda_{E}=i \ell \nu$, with $\ell=0, \pm 1, \pm 2, \ldots$. Each eigenspace at $\lambda_{\mathrm{E}}$ is infinitely degenerate, and the corresponding left and right eigenelements are, for instance, $\check{\mu}_{n}^{\ell}$ $=|n+\ell\rangle\langle n|$ and $\mu_{n}^{\ell}=|n\rangle\langle n+\ell|$. These eigenelements constitute a complete and orthonormal basis over the eigenspace at this eigenvalue. In particular, the projector over the eigenspace at $\lambda_{\mathrm{E}}=i \ell \nu$ is defined on an operator $X$ as

$$
\mathcal{U}^{\lambda_{\mathrm{E}}=i \ell \nu} X=\sum_{n} \mu_{n}^{\ell} \operatorname{Tr}_{\mathrm{E}}\left\{\check{\mu}_{n}^{\ell} X\right\}=\sum_{n}|n\rangle\langle n|X| n+\ell\rangle\langle n+\ell|,
$$

where $\operatorname{Tr}_{\mathrm{E}}$ denotes the trace over the external degrees of freedom.

At higher orders in the expansion in $\eta$, internal and external degrees of freedom are coupled and the degeneracy of the subspaces at eigenvalue $\lambda_{\mathrm{E}}$ is lifted $[19,22]$. The perturbative corrections to the eigenvalues $\lambda_{0}$ to the eigenelements $\rho_{0}^{\lambda}$ and $\check{\rho}_{0}^{\lambda}$ and to the projectors $\mathcal{P}_{0}^{\lambda}$ are found by solving iteratively the secular equations at the same order in the perturbative expansion and are given by

$$
\left(1-\mathcal{P}_{0}^{\lambda}\right) \varrho_{1}^{\lambda}=-\frac{1-\mathcal{P}_{0}^{\lambda}}{\lambda_{0}-\mathcal{L}_{0}}\left(\lambda_{1}-\mathcal{L}_{1}\right) \varrho_{0}^{\lambda},
$$

where $\mathcal{P}_{0}^{\lambda}$ is the zeroth-order projector onto the subspace at eigenvalue $\lambda, \mathcal{P}_{0}^{\lambda}=\varrho_{0}^{\lambda} \otimes \check{\varrho}_{0}^{\lambda}$. Using Eq. (17) we obtain

$$
\begin{aligned}
\left(1-\mathcal{P}_{0}^{\lambda}\right) \varrho_{2}^{\lambda}= & -\frac{1-\mathcal{P}_{0}^{\lambda}}{\lambda_{0}-\mathcal{L}_{0}}\left[-\left(\lambda_{1}-\mathcal{L}_{1}\right) \frac{1-\mathcal{P}_{0}^{\lambda}}{\lambda_{0}-\mathcal{L}_{0}}\left(\lambda_{1}-\mathcal{L}_{1}\right)+\left(\lambda_{2}\right.\right. \\
& \left.\left.-\mathcal{L}_{2}\right)\right] \varrho_{0}^{\lambda}
\end{aligned}
$$

Analogously, one finds the perturbative corrections to the left eigenelements $\check{\varrho}_{0}^{\lambda}$. This in turn allows one to evaluate the perturbative corrections to the projectors $\mathcal{P}_{0}^{\lambda}$, which are immediately found by using the explicit form of $\check{\varrho}_{j}^{\lambda}$ and $\varrho_{j}^{\lambda}$ into the relations

$$
\begin{gathered}
\mathcal{P}_{1}^{\lambda}=\varrho_{0}^{\lambda} \check{\varrho}_{1}^{\lambda}+\varrho_{1}^{\lambda} \check{\varrho}_{0}^{\lambda}, \\
\mathcal{P}_{2}^{\lambda}=\varrho_{0}^{\lambda} \check{\varrho}_{2}^{\lambda}+\varrho_{1}^{\lambda} \check{\varrho}_{1}^{\lambda}+\varrho_{2}^{\lambda} \check{\varrho}_{0}^{\lambda} .
\end{gathered}
$$

The equations for the corrections $\lambda_{1}$ and $\lambda_{2}$ to $\lambda_{0}$ are

$$
\begin{gathered}
\lambda_{1}=\operatorname{Tr}\left\{\check{\varrho}_{0}^{\lambda} \mathcal{L}_{1} \varrho_{0}^{\lambda}\right\}=0, \\
\lambda_{2}=\operatorname{Tr}\left\{\check{\varrho}_{0}^{\lambda} \mathcal{L}_{2} \rho_{0}^{\lambda}\right\}+\operatorname{Tr}\left\{\check{\rho}_{0}^{\lambda} \mathcal{L}_{1} \rho_{1}^{\lambda}\right\}=\operatorname{Tr}\left\{\check{\rho}_{0}^{\lambda} \mathcal{L}_{2} \rho_{0}^{\lambda}\right\} \\
+\operatorname{Tr}\left\{\check{\rho}_{0}^{\lambda} \mathcal{L}_{1} \frac{1-\mathcal{P}_{0}^{\lambda}}{\lambda_{0}-\mathcal{L}_{0}} \mathcal{L}_{1} \rho_{0}^{\lambda}\right\},
\end{gathered}
$$

where we have used the relation $\mathcal{P}_{0}^{\lambda} \mathcal{L}_{1} \mathcal{P}_{0}^{\lambda}=0$ in Eq. (21).

A relevant eigenelement of this spectral decomposition is the one at $\lambda_{0}=0$ : namely, the steady-state density matrix. At zero order this is given by $\varrho_{0}^{\lambda=0}=\rho_{0} \mu$, where $\mu$ is the density matrix for the external degrees of freedom in the final stage of the laser-cooling dynamics and has the form

$$
\mu=\frac{1}{1+\langle n\rangle}\left(\frac{\langle n\rangle}{1+\langle n\rangle}\right)^{a^{\dagger} a}
$$

and

$$
\langle n\rangle=\operatorname{Tr}\left\{a^{\dagger} a \mu\right\}
$$

is the average phonon number at steady state. Correspondingly, $\check{\varrho}_{0}^{\lambda=0}=\mathbb{1}_{\mathrm{I}} \mathbb{1}_{\mathrm{E}}$, where $1_{j}$ is the identity for the Hilbert space of the internal $(j=\mathrm{I})$ and the external $(j=\mathrm{E})$ degrees of freedom.

\section{B. Explicit form of the sidebands of the elastic and inelastic components}

We now proceed in evaluating the spectrum of resonance fluorescence using the terms introduced in the previous section. At zero order in the mechanical effects, the spectrum is

$$
S_{0}(\omega)=\tilde{\chi}(\psi) \operatorname{Re} \sum_{\lambda_{0}} \frac{1}{\mathrm{i}\left(\omega-\omega_{\mathrm{L}}\right)-\lambda} G(\lambda)
$$

where $G(\lambda)=\operatorname{Tr}\left\{D_{0}^{\dagger} \mathcal{P}_{0}^{\lambda} D_{0} \rho_{0}\right\}$, which corresponds to the spectrum of the bare atomic dipole. It thus exhibit peaks which are located at frequencies equal to the real part of the eigenvalues $\lambda_{\mathrm{I}}$, whose width is determined by the imaginary part of $\lambda_{\text {I }}$. Note that $\operatorname{Tr}\left\{D_{0}^{\dagger} \mathcal{P}_{0}^{\lambda} D_{0} \rho_{0}\right\}=\operatorname{Tr}\left\{\sigma^{\dagger} \rho_{0}^{\lambda_{1}}\right\} \operatorname{Tr}\left\{\tilde{\rho}_{0}^{\lambda_{I}} \sigma \rho_{0}\right\}$. For later convenience, we introduce the scalar functions

$$
\begin{gathered}
g\left(\lambda_{\mathrm{I}}\right)=\operatorname{Tr}\left\{\sigma^{\dagger} \rho_{0}^{\lambda_{\mathrm{I}}}\right\}, \\
\check{g}\left(\lambda_{\mathrm{I}}\right)=\operatorname{Tr}\left\{\check{\rho}_{0}^{\lambda_{\mathrm{I}}} \sigma \rho_{0}\right\},
\end{gathered}
$$

such that with this notation $G(\lambda)=g\left(\lambda_{\mathrm{I}}\right) \check{g}\left(\lambda_{\mathrm{I}}\right)$.

The term $S_{1}(\omega)=0$, since the motional steady state $\mu$, Eq. (23), is a thermal state and thus diagonal in the vibrational number basis [12].

The features due to the mechanical effects thus manifest at second order in the Lamb-Dicke expansion, and the corresponding term has the form

$$
S_{2}(\omega)=\tilde{\chi}(\psi) \operatorname{Re} \sum_{\lambda} \frac{1}{\mathrm{i}\left(\omega-\omega_{\mathrm{L}}\right)-\lambda} F(\lambda),
$$

where

$$
F(\lambda)=\sum_{a+b+c+d=2} \operatorname{Tr}\left\{D_{a}^{\dagger} \mathcal{P}_{b}^{\lambda} D_{c} \rho_{d}\right\} .
$$

The function $F(\lambda)$ can be decomposed into three contributions: namely,

$$
F\left(\lambda_{\mathrm{E}}, \lambda_{\mathrm{I}}\right)=F_{0}\left(\lambda_{\mathrm{I}}\right) \delta_{\lambda_{\mathrm{E}}, 0}+F_{+}\left(\lambda_{\mathrm{I}}\right) \delta_{\lambda_{\mathrm{E}}, i \nu}+F_{-}\left(\lambda_{\mathrm{I}}\right) \delta_{\lambda_{\mathrm{E}},-i \nu}
$$

This decomposition allows one to identify the effects of the quantum motion on the features of the spectrum. The term 
$F_{0}\left(\lambda_{\mathrm{I}}\right)$ is a second-order correction to the features of the zeroth-order spectrum, $S_{0}(\omega)$. Namely, it gives rise to small corrections to the curves of the zeroth-order spectrum; however, it does not affect relevantly its general form. The terms
$F_{ \pm}\left(\lambda_{\mathrm{I}}\right)$, on the other hand, give rise to novel peaks centered at the frequencies $\operatorname{Im}\left\{\lambda_{\mathrm{I}}\right\} \pm \nu$, which can be identified with the sidebands of the peaks appearing in $S_{0}(\omega)$. These terms have the form

$$
\begin{aligned}
F_{+}\left(\lambda_{\mathrm{I}}\right)= & \eta^{2} \cos ^{2} \theta r\left(\lambda_{\mathrm{I}}, i \nu\right)\left[\left\{r_{*}\left(\lambda_{\mathrm{I}}, i \nu\right)+u\left(\lambda_{\mathrm{I}}, i \nu\right)\right\}\langle n\rangle-t\left(\lambda_{\mathrm{I}}, i \nu\right)\right] \\
& -\eta^{2} \cos \theta \cos \psi\left(g\left(\lambda_{\mathrm{I}}\right)\left[\left\{r_{*}\left(\lambda_{\mathrm{I}}, i \nu\right)+u\left(\lambda_{\mathrm{I}}, i \nu\right)\right\}\langle n\rangle-t\left(\lambda_{\mathrm{I}}, i \nu\right)\right]+r\left(\lambda_{\mathrm{I}}, i \nu\right) \check{g}\left(\lambda_{\mathrm{I}}\right)\langle n\rangle\right) \\
& +\eta^{2} \cos ^{2} \psi g\left(\lambda_{\mathrm{I}}\right) \check{g}\left(\lambda_{\mathrm{I}}\right)\langle n\rangle
\end{aligned}
$$

and

$$
\begin{aligned}
F_{-}\left(\lambda_{\mathrm{I}}\right)= & \eta^{2} \cos ^{2} \theta r\left(\lambda_{\mathrm{I}},-i \nu\right)\left[\left\{r_{*}\left(\lambda_{\mathrm{I}},-i \nu\right)+u\left(\lambda_{\mathrm{I}},-i \nu\right)\right\}(\langle n\rangle+1)+t\left(\lambda_{\mathrm{I}},-i \nu\right)\right] \\
& -\eta^{2} \cos \theta \cos \psi\left(g\left(\lambda_{\mathrm{I}}\right)\left[\left\{r_{*}\left(\lambda_{\mathrm{I}},-i \nu\right)+u\left(\lambda_{\mathrm{I}},-i \nu\right)\right\}(\langle n\rangle+1)+t\left(\lambda_{\mathrm{I}},-i \nu\right)\right]+r\left(\lambda_{\mathrm{I}},-i \nu\right) \check{g}\left(\lambda_{\mathrm{I}}\right)(\langle n\rangle+1)\right) \\
& +\eta^{2} \cos ^{2} \psi g\left(\lambda_{\mathrm{I}}\right) \check{g}\left(\lambda_{\mathrm{I}}\right)(\langle n\rangle+1),
\end{aligned}
$$

where we have used $\eta=k x_{0}$ with $x_{0}=\sqrt{\hbar / 2 m \nu}$ and we have introduced the functions

$$
\begin{aligned}
& r\left(\lambda_{\mathrm{I}}, \lambda_{\mathrm{E}}\right)=\frac{1}{\hbar} \operatorname{Tr}_{\mathrm{I}}\left\{D_{0}^{\dagger}\left(\lambda_{\mathrm{I}}+\lambda_{\mathrm{E}}-\mathcal{L}_{\mathrm{I}}\right)^{-1}\left[V_{1}, \rho_{0}^{\lambda_{\mathrm{I}}}\right]\right\}, \\
& r_{*}\left(\lambda_{\mathrm{I}}, \lambda_{\mathrm{E}}\right)=\frac{1}{\hbar} \operatorname{Tr}_{\mathrm{I}}\left\{\check{\check{\rho}}_{0}^{\lambda} D_{0}\left(\lambda_{\mathrm{E}}+\mathcal{L}_{\mathrm{I}}\right)^{-1}\left[V_{1}, \rho_{0}\right]\right\}, \\
& u\left(\lambda_{\mathrm{I}}, \lambda_{\mathrm{E}}\right)=-\frac{1}{\hbar} \operatorname{Tr}_{\mathrm{I}}\left\{\check{\rho}_{0}^{\lambda} \lambda_{\mathrm{I}}\left[V_{1},\left(\lambda_{\mathrm{I}}+\lambda_{\mathrm{E}}-\mathcal{L}_{\mathrm{I}}\right)^{-1} D_{0} \rho_{0}\right]\right\}, \\
& t\left(\lambda_{\mathrm{I}}, \lambda_{\mathrm{E}}\right)=\frac{1}{\hbar} \operatorname{Tr}_{\mathrm{I}}\left\{\stackrel{\check{\rho}}{\rho_{0}^{\lambda}} D_{0}\left(\lambda_{\mathrm{E}}+\mathcal{L}_{\mathrm{I}}\right)^{-1} \rho_{0} V_{1}\right\} \\
& -\frac{1}{\hbar} \operatorname{Tr}_{\mathrm{I}}\left\{V_{1} \check{\rho}_{0}^{\lambda}\left(\lambda_{\mathrm{E}}+\lambda_{\mathrm{I}}-\mathcal{L}_{\mathrm{I}}\right)^{-1} D_{0} \rho_{0}\right\} .
\end{aligned}
$$

The basic steps that lead to Eqs. (29)-(34) are reported in Appendix A. The results are plotted and discussed for some parameter regimes in Sec. II.

We now discuss some general properties of the equations for two cases: when the laser, driving the dipole and cooling the motion, is a traveling wave and when it is a standing wave.

Traveling-wave drive. The case when the driving laser is a traveling wave is found by setting $\zeta^{\prime}(\varphi)=i$ at $\varphi=0$ in Eq. (13). We evaluate the terms (31)-(34) using the zeroth-order eigenelements $\rho_{0}^{\lambda}$ and $\check{\rho}_{0}^{\lambda}$ determined by the eigenvalue equations $\mathcal{L}_{0} \rho_{\mathrm{I}}^{\lambda}=\lambda_{0} \rho_{\mathrm{I}}^{\lambda}$ and $\check{\rho}_{\mathrm{I}}^{\lambda} \mathcal{L}_{0}=\lambda_{0} \check{\rho}_{\mathrm{I}}^{\lambda}$, as in [16]. The eigenvalue $\lambda_{\mathrm{I}}=0$ gives the contribution of the elastic peak. As we focus on the case of a dipole driven at saturation, the three othernonvanishing - eigenvalues of the zeroth-order spectrum determine the position and widths of the Mollow peaks.

We characterize the higher-order contributions to the spectrum by their corresponding eigenvalues at zero order and analyze the single terms composing $S_{2}(\omega)$, Eq. (26). As
$S_{0}(\omega)$ never vanishes for traveling-wave drives, the term $F_{0}$ gives rise to small corrections to the elastic peak (component at $\lambda_{\mathrm{I}}=0$ ) and to the Mollow spectrum (components at $\lambda_{\mathrm{I}}$ $\neq 0)$. The terms $F_{ \pm}(\lambda)$ correspond to novel peaks which add to the ones of the zeroth-order spectrum. At $\lambda_{I}=0$ these terms give rise to the motional sidebands of the elastic peak, centered about $\lambda_{\mathrm{E}}= \pm i \nu$. In this case the terms (31)-(34) have to be evaluated for $\check{\rho}_{0}^{\lambda_{\mathrm{I}}}=\mathbb{1}_{\mathrm{I}}$ and $\rho_{0}^{\lambda_{\mathrm{I}}}=\rho_{0}$. By applying the cyclic properties of the trace one finds that $u\left(0, \lambda_{\mathrm{E}}\right)=0$ and that $r_{*}\left(0, \lambda_{\mathrm{E}}\right)=r\left(0, \lambda_{\mathrm{E}}\right)^{*}$. Since $\lambda_{\mathrm{I}}=0$, the width and shape of these curves are critically determined by the second-order correction $\lambda_{2}$ to the eigenvalue. The explicit evaluation can be found in $[12,22]$. The result we find is in agreement with the one reported in [10].

The mechanical effects on the inelastic part of the spectrum manifest themselves in the components at $\lambda_{\mathrm{I}} \neq 0$. They give rise to small shifts of the center frequency $\operatorname{Im}\left\{\lambda_{\mathrm{I}}\right\}$ and of the linewidth $\operatorname{Re}\left\{\lambda_{\mathrm{I}}\right\}$, and to novel peaks which are centered at the frequencies $\operatorname{Im} \lambda_{\mathrm{I}} \pm \nu$ and which we discuss in the following. These peaks are the sidebands of the corresponding zeroth-order curves in the Mollow spectrum. Their width is the width of the corresponding zeroth-order Mollow peak: namely, $\operatorname{Re}\left\{\lambda_{\mathrm{I}}\right\}$. The intensity of the particular signals is proportional to $F_{ \pm}\left(\lambda_{\mathrm{I}}\right)$, where generally all the terms (31)-(34), $g\left(\lambda_{\mathrm{I}}\right)$, and $\check{g}\left(\lambda_{\mathrm{I}}\right)$ contribute. Their shape is in general a superposition of Lorentz [real part of $\left.F_{ \pm}\left(\lambda_{\mathrm{I}}\right)\right]$ and Fano-like [imaginary part of $\left.F_{ \pm}\left(\lambda_{\mathrm{I}}\right)\right]$ curves. Note that in the plots shown in Sec. II, the real part of $F_{ \pm}\left(\lambda_{\mathrm{I}}\right)$ dominates for the visible peaks and thus these sidebands appear as Lorentz curves in the spectrum. Their visibility, however, depends on the detection angle. Indeed, also for these terms there are interference effects that give rise to an asymmetry between the right and left sidebands of a given inelastic peak. Note that the sidebands of the central inelastic peak are located at the same frequencies of the Stokes and anti-Stokes sidebands. These terms do not vanish, but are usually hidden by the Stokes and anti-Stokes contributions. Spectra for different parameter regimes are shown in Fig. 2. 
Standing-wave drive. The case when the driving laser is a standing wave is found by setting $\zeta(\varphi)=\cos \varphi$ in $V_{0}$ and $\zeta^{\prime}(\varphi)=-\sin \varphi$ in Eq. (13). The general characteristic of the terms composing the spectrum is basically the same as for a traveling wave; the difference arises mainly in the dependence of the curves on the scattering angle. The analytical form of the two sidebands, which we obtain by evaluating explicitly $F_{ \pm}(0)$ and the linewidth $\lambda_{2}$ of the resonances, is in agreement with the formulas reported in [10]. A peculiar behavior is found if the atom is placed at the node of the standing wave, $\zeta(\varphi)=0$, which we discuss in the following. Indeed, in the node of a standing wave the electromagnetic field vanishes at zero order in the Lamb-Dicke expansion. Hence, the dipole is excited by processes at higher order in the Lamb-Dicke parameter $\eta$. In other words, in this regime the spectrum of resonance fluorescence is only due to the gradient of the electromagnetic field over the finite size of the ion wave packet and thus originates from mechanical effects of the atom-photon interaction. In the formulas the terms $V_{0}=V_{2}=0$ and the steady-state density matrix $\rho_{0}$ $=|g\rangle\langle g| \mu$. Details of the eigenelements and eigenvalues of $\mathcal{L}_{\mathrm{I}}$ are reported in Appendix B. In this case $D_{0} \rho_{0}=0$ and consequently the term $u(\lambda)=0$; see Eq. (33). For the same reason the second term on the right-hand side (RHS) of $t(\lambda)$ vanishes; see Eq. (34). Also the first term on the RHS of $t(\lambda)$ vanishes, as one can verify by taking the explicit form of $V_{1}$, Eq. (13), and of the eigenelements. Hence $t(\lambda)=0$. Moreover, in Appendix B we show that $F_{0}(\lambda)=0$. The only terms which do not vanish are $r\left(0, \lambda_{\mathrm{E}}\right)$ and $r_{*}\left(0, \lambda_{\mathrm{E}}\right)$ in $F_{ \pm}(\lambda)$, Eqs. (31) and (32). Therefore, the spectrum is at second order in the Lamb-Dicke expansion, and the only nonvanishing contributions are the Stokes and anti-Stokes sidebands of the elastic peak. Spectra evaluated for different parameters are shown in Figs. 3 and 4.

\section{CONCLUSIONS}

The spectrum of resonance fluorescence of a cold trapped ion, which is in the stationary state of laser cooling, presents several features due to the mechanical effects of light on the quantum motion of the atomic center of mass. In this work we have shown that, in addition to the well-known Stokes and anti-Stokes components, similar features appear also in the inelastic spectrum. These features are sidebands of the inelastic peaks and have no classical analog. The quantum features in the mechanical effects of atom-photon interaction become visible at low temperatures, when the motional state is close to the ground state and when the transition linewidth is smaller than the trap frequency: namely, in the regime where the ion can be sideband cooled. This parameter regime can be encountered, for instance, in experimental situations like [13].

In our investigation we have considered the light scattered from a laser which sideband-cools the motion in two cases: when the drive is a traveling wave and when it is a standing wave. A peculiar behavior is observed when the drive is a standing wave: Here, depending on the position of the ion in the mode, the spectrum changes dramatically. In particular, in the node of the standing wave we find that the spectrum is solely composed by the Stokes and anti-Stokes sidebands, while any other contribution is at higher order in the LambDicke expansion. In this regime we recover the form of the sidebands components as predicted in [10]. The situation in the node can be compared with a $\Lambda$ system driven at twophoton resonance, where the zeroth-order contribution vanishes due to the existence of the dark-state coherence. In that case, however, sidebands and unshifted contributions of the inelastic spectrum are visible, since the electric field does not vanish at zero order in the Lamb-Dicke expansion [12].

In conclusion, we have studied the effects of the quantum center-of-mass motion on the inelastic part of the spectrum. This work complements previous theoretical analyses $[9,10]$, which investigated the elastic part. In the future we will look at how the quantum center-of-mass motion affects higherorder correlation functions of the scattered light.

\section{ACKNOWLEDGMENTS}

M.B. and G.M. acknowledge hospitality from the Centro Internacional de Ciencia in Cuernavaca, Mexico during the Humboldtkolleg "Low dimensional systems in quantum optics," where part of this work has been done. The authors are grateful to Gloria Koenigsberger for pivotal support. M.B. is supported by the Alexander von Humboldt foundation. W.M. acknowledges financial support by the Landesstiftung Baden-Württemberg. G.M. is supported by the Spanish Ministerio de Educaccion y Ciencia (Ramon-y-Cajal).

\section{APPENDIX A: EVALUATION OF $F_{ \pm}(\lambda)$}

The terms of Eq. (28) which contribute to $F_{ \pm}(\lambda)$ are

$$
\begin{gathered}
f_{1}(\lambda)=\operatorname{Tr}\left\{D_{0}^{\dagger} \mathcal{P}_{1}^{\lambda} D_{0} \varrho_{1}\right\}+\operatorname{Tr}\left\{D_{0}^{\dagger} \mathcal{P}_{2}^{\lambda} D_{0} \varrho_{0}\right\}, \\
f_{2}(\lambda)=\operatorname{Tr}\left\{D_{1}^{\dagger} \mathcal{P}_{0}^{\lambda} D_{0} \varrho_{1}\right\}+\operatorname{Tr}\left\{D_{1}^{\dagger} \mathcal{P}_{1}^{\lambda} D_{0} \varrho_{0}\right\} \\
f_{3}(\lambda)=\operatorname{Tr}\left\{D_{0}^{\dagger} \mathcal{P}_{1}^{\lambda} D_{1} \varrho_{0}\right\} \\
f_{4}(\lambda)=\operatorname{Tr}\left\{D_{1}^{\dagger} \mathcal{P}_{0}^{\lambda} D_{1} \varrho_{0}\right\}
\end{gathered}
$$

We evaluate now their explicit form using the perturbative expansion using the definitions introduced in Sec. III. We denote by the superscript \pm the part of the term which contributes to $F_{ \pm}(\lambda)$. The relevant part of the first term can be rewritten as

$$
\begin{aligned}
f_{1}^{ \pm}(\lambda)= & \frac{k^{2} \cos ^{2} \theta}{\hbar} r\left(\lambda_{\mathrm{I}}, \lambda_{\mathrm{E}}\right)\left[\operatorname { T r } _ { \mathrm { E } } \{ x \mathcal { U } ^ { \lambda } \mathrm { E } x \mu \} \left(\operatorname { T r } _ { \mathrm { I } } \left\{\check { \rho } _ { 0 } ^ { \lambda _ { \mathrm { I } } } D _ { 0 } \left(\lambda_{\mathrm{E}}\right.\right.\right.\right. \\
& \left.\left.\left.+\mathcal{L}_{\mathrm{I}}\right)^{-1}\left[V_{1}, \rho_{0}\right]\right\}-\operatorname{Tr}_{\mathrm{I}}\left\{\check{\rho}_{0}^{\lambda}\left[V_{1},\left(\lambda_{\mathrm{I}}+\lambda_{\mathrm{E}}-\mathcal{L}_{\mathrm{I}}\right)^{-1} D_{0} \rho_{0}\right]\right\}\right) \\
& +\operatorname{Tr}_{\mathrm{E}}\left\{x \mathcal{U} \mathcal{U}^{\lambda} \mathrm{E}[x, \mu]\right\}\left(\operatorname{Tr}_{\mathrm{I}}\left\{\check{\rho}_{0}^{\lambda} D_{0}\left(\lambda_{\mathrm{E}}+\mathcal{L}_{\mathrm{I}}\right)^{-1} \rho_{0} V_{1}\right\}\right. \\
& \left.\left.-\operatorname{Tr}_{\mathrm{I}}\left\{V_{1} \check{\rho}_{0}^{\lambda_{\mathrm{I}}}\left(\lambda_{\mathrm{I}}+\lambda_{\mathrm{E}}-\mathcal{L}_{\mathrm{I}}\right)^{-1} D_{0} \rho_{0}\right\}\right)\right],
\end{aligned}
$$

where we have used the function $r\left(\lambda_{\mathrm{I}}, \lambda_{\mathrm{E}}\right)$ defined in Eq. (31). Note that for $\lambda_{I}=0$ it reduces to the function $-r\left(\lambda_{E}\right)$ in [10]. Using definitions (32)-(34) we obtain 


$$
\begin{aligned}
f_{1}^{ \pm}(\lambda)= & k^{2} \cos ^{2} \operatorname{\theta r}\left(\lambda_{\mathrm{I}}+\lambda_{\mathrm{E}}\right)\left[\left\{r_{*}\left(\lambda_{\mathrm{I}}, \lambda_{\mathrm{E}}\right)\right.\right. \\
& \left.\left.+u\left(\lambda_{\mathrm{I}}, \lambda_{\mathrm{E}}\right)\right\} \operatorname{Tr}_{\mathrm{E}}\left\{x \mathcal{U}^{\lambda} \mathrm{E} x \mu\right\}+t\left(\lambda_{\mathrm{I}}, \lambda_{\mathrm{E}}\right) \operatorname{Tr}_{\mathrm{E}}\left\{x \mathcal{U}^{\lambda} \mathrm{E}[x, \mu]\right\}\right] .
\end{aligned}
$$

It can be simply verified that function $r_{*}\left(\lambda_{\mathrm{I}}, \lambda_{\mathrm{E}}\right)$ in Eq. (32) fulfills the relation $r_{*}\left(0, \lambda_{\mathrm{E}}\right)=r\left(0, \lambda_{\mathrm{E}}\right)^{*}$. Moreover, the term $u\left(0, \lambda_{\mathrm{E}}\right)=0$. Finally, the function $t\left(\lambda_{\mathrm{I}}, \lambda_{\mathrm{E}}\right)$, defined in Eq. (31), reduces for $\lambda_{\mathrm{I}}=0$ to the function $t\left(\lambda_{\mathrm{E}}\right)$ in [10]. as

Using these definitions, the other terms can be rewritten

$$
\begin{aligned}
f_{2}^{ \pm}(\lambda)= & -k^{2} \cos \theta \cos \psi g\left(\lambda_{\mathrm{I}}\right)\left[\left\{r_{*}\left(\lambda_{\mathrm{I}}, \lambda_{\mathrm{E}}\right)\right.\right. \\
& \left.\left.+u\left(\lambda_{\mathrm{I}}, \lambda_{\mathrm{E}}\right)\right\} \operatorname{Tr}_{\mathrm{E}}\left\{x \mathcal{U}^{\lambda} \mathrm{E} x \mu\right\}+t\left(\lambda_{\mathrm{I}}, \lambda_{\mathrm{E}}\right) \operatorname{Tr}_{\mathrm{E}}\left\{x \mathcal{U}^{\lambda} \mathrm{E}[x, \mu]\right\}\right]
\end{aligned}
$$

and

$$
\begin{gathered}
f_{3}^{ \pm}(\lambda)=-k^{2} \cos \theta \cos \psi \check{g}\left(\lambda_{\mathrm{I}}\right) r\left(\lambda_{\mathrm{I}}, \lambda_{\mathrm{E}}\right) \operatorname{Tr}_{\mathrm{E}}\left\{x \mathcal{U}^{\lambda} \mathrm{E} x \mu\right\}, \\
f_{4}^{ \pm}(\lambda)=k^{2} \cos ^{2} \psi g\left(\lambda_{\mathrm{I}}\right) \check{g}\left(\lambda_{\mathrm{I}}\right) \operatorname{Tr}_{\mathrm{E}}\left\{x \mathcal{U}^{\lambda_{\mathrm{E}}} x \mu\right\},
\end{gathered}
$$

where we have used the definitions from Eq. (26). The trace terms over the external degrees of freedom are conveniently evaluated using the basis set corresponding to the projectors in Eq. (16), giving

$$
\begin{gathered}
\operatorname{Tr}_{\mathrm{E}}\left\{x \mathcal{U}^{\lambda} \mathrm{E}_{\mathrm{E}} x\right\}=x_{0}^{2}\left[\delta_{\lambda_{\mathrm{E}},-i \nu}(\langle n\rangle+1)+\delta_{\lambda_{\mathrm{E}}, i \nu}\langle n\rangle\right], \\
\operatorname{Tr}_{\mathrm{E}}\left\{x \mathcal{U}^{\lambda} \mathrm{E}[x, \mu]\right\}=x_{0}^{2}\left[-\delta_{\lambda_{\mathrm{E}}, i \nu}+\delta_{\lambda_{\mathrm{E}},-i \nu}\right],
\end{gathered}
$$

with $x_{0}=\sqrt{\hbar / 2 M \nu}$. Using these results in Eq. (28) we obtain Eqs. (29) and (30).

\section{APPENDIX B: IONS IN THE NODE OF THE STANDING WAVE}

In this appendix we evaluate explicitly the contribution $F_{0}\left(\lambda_{\mathrm{I}}\right)$ and show that it identically vanishes in the case where the atom is at the node of the standing wave.

Let us first discuss the terms contributing to $F_{0}\left(\lambda_{\mathrm{I}}\right)$. In the node of the standing wave $\rho_{0}=|g\rangle\langle g|$. By using the relation $D_{a} \rho_{0}=0 \quad(a=0,1,2, \ldots)$, the terms which do not trivially vanish are

$$
F_{0}\left(\lambda_{I}\right)=f_{1}^{0}\left(\lambda_{\mathrm{I}}\right)+f_{5}^{0}\left(\lambda_{\mathrm{I}}\right)+f_{6}^{0}\left(\lambda_{\mathrm{I}}\right),
$$

where $f_{j}^{0}$ are the components of the terms

$$
\begin{aligned}
& f_{1}\left(\lambda_{\mathrm{I}}\right)=\operatorname{Tr}\left\{D_{0}^{\dagger} \mathcal{P}_{1}^{\lambda} D_{0} \varrho_{1}\right\}, \\
& f_{5}\left(\lambda_{\mathrm{I}}\right)=\operatorname{Tr}\left\{D_{0}^{\dagger} \mathcal{P}_{0}^{\lambda} D_{0} \varrho_{2}\right\}, \\
& f_{6}\left(\lambda_{\mathrm{I}}\right)=\operatorname{Tr}\left\{D_{0}^{\dagger} \mathcal{P}_{0}^{\lambda} D_{1} \varrho_{1}\right\},
\end{aligned}
$$

which contribute to $F_{0}\left(\lambda_{\mathrm{I}}\right)$. Their explicit form is found with the internal eigenelements, which at the node take the form

$$
\rho^{\text {st }}=|g\rangle\langle g|, \quad \check{\rho}^{\text {st }}=\mathbb{1}_{\mathrm{E}}
$$

$$
\begin{gathered}
\rho^{0}=|e\rangle\langle e|-| g\rangle\left\langle g\left|, \quad \check{\rho}^{0}=\right| e\right\rangle\langle e|, \\
\rho^{+}=|e\rangle\left\langle g\left|, \quad \check{\rho}^{+}=\right| g\right\rangle\langle e|, \\
\rho^{-}=|g\rangle\left\langle e\left|, \quad \check{\rho}^{-}=\right| e\right\rangle\langle g|,
\end{gathered}
$$

with the corresponding eigenvalues $\lambda^{\text {st }}=0, \lambda_{\mathrm{I}}^{0}=-\gamma$, and $\lambda_{\mathrm{I}}^{ \pm}$ $=-\gamma / 2 \pm i \delta$. Substituting into Eq. (B4) one finds $f_{6}^{0}(\lambda)=0$. The other two terms give, at $\lambda_{\mathrm{E}}=0$,

$$
\begin{gathered}
f_{1}^{0}\left(\lambda_{\mathrm{I}}\right)=-\delta_{\lambda_{\mathrm{I}}, \lambda^{-}} \frac{\Omega^{2} k^{2} \cos ^{2} \theta}{4} \sum_{\lambda_{\mathrm{E}}^{\prime}} \frac{\operatorname{Tr}_{\mathrm{E}}\left\{x \mathcal{U}^{\lambda_{\mathrm{E}}^{\prime}} x \mu\right\}}{\left(\lambda^{-}-\lambda_{\mathrm{E}}^{\prime}\right)\left(\lambda^{+}+\lambda_{\mathrm{E}}^{\prime}\right)}, \\
f_{5}^{0}\left(\lambda_{\mathrm{I}}\right)=\delta_{\lambda_{\mathrm{I}}, \lambda^{-}} \frac{\Omega^{2} k^{2} \cos ^{2} \theta}{4} \sum_{\lambda_{\mathrm{E}}^{\prime}}\left[\frac{\operatorname{Tr}_{\mathrm{E}}\left\{x \mathcal{U}^{\lambda_{\mathrm{E}}^{\prime}} x \mu\right\}}{\lambda^{0}\left(\lambda^{+}+\lambda_{\mathrm{E}}^{\prime}\right)}\right. \\
\left.+\frac{\operatorname{Tr}_{\mathrm{E}}\left\{x \mathcal{U}^{\lambda_{\mathrm{E}}^{\prime}} \mu x\right\}}{\lambda^{0}\left(\lambda^{-}+\lambda_{\mathrm{E}}^{\prime}\right)}\right] .
\end{gathered}
$$

Using the expressions for the external traces, Eqs. (A1) and (A2), and the explicit form of eigenelements and eigenvectors, one finds that $f_{1}^{0}\left(\lambda_{\mathrm{I}}\right)=-f_{5}^{0}\left(\lambda_{\mathrm{I}}\right)$. Thus, the two terms mutually cancel in Eq. (B1) and hence $F_{0}\left(\lambda_{\mathrm{I}}\right)=0$.
[1] For a recent review, see D. Leibfried, R. Blatt, C. Monroe, and D. Wineland Rev. Mod. Phys. 75, 281 (2003), and references therein.

[2] P. S. Jessen, C. Gerz, P. D. Lett, W. D. Phillips, S. L. Rolston, R. J. C. Spreeuw, and C. I. Westbrook, Phys. Rev. Lett. 69, 49 (1992); M. Gatzke, G. Birkl, P. S. Jessen, A. Kastberg, S. L. Rolston, and W. D. Phillips, Phys. Rev. A 55, R3987 (1997).

[3] J. T. Höffges, H. W. Baldauf, T. Eichler, S. R. Helmfrid, and H. Walther, Opt. Commun. 133, 170 (1997); J. T. Höffges, H. W. Baldauf, W. Lange, and H. Walther, J. Mod. Opt. 44, 1999 (1997).

[4] Ch. Raab, J. Eschner, J. Bolle, H. Oberst, F. Schmidt-Kaler, and R. Blatt, Phys. Rev. Lett. 85, 538 (2000).

[5] V. Bühner and Chr. Tamm, Phys. Rev. A 61, 061801(R) (2000).
[6] M. A. Wilson, P. Bushev, J. Eschner, F. Schmidt-Kaler, C. Becher, R. Blatt, and U. Dorner, Phys. Rev. Lett. 91, 213602 (2003).

[7] P. Bushev, A. Wilson, J. Eschner, C. Raab, F. Schmidt-Kaler, C. Becher, and R. Blatt, Phys. Rev. Lett. 92, 223602 (2004).

[8] P. Bushev, D. Rotter, A. Wilson, F. Dubin, Ch. Becher, J. Eschner, R. Blatt, V. Steixner, P. Rabl, and P. Zoller, Phys. Rev. Lett. 96, 043003 (2006).

[9] M. Lindberg, Phys. Rev. A 34, 3178 (1986).

[10] J. I. Cirac, R. Blatt, A. S. Parkins, and P. Zoller, Phys. Rev. A 48, 2169 (1993).

[11] G. C. Hegerfeldt and M. B. Plenio, Phys. Rev. A 52, 3333 (1995).

[12] M. Bienert, W. Merkel, and G. Morigi, Phys. Rev. A 69, 013405 (2004). See also M. Bienert, Ph.D. thesis, University 
of Ulm, 2004.

[13] Th. Becker, J. v. Zanthier, A. Yu. Nevsky, Ch. Schwedes, M. N. Skvortsov, H. Walther, and E. Peik, Phys. Rev. A 63, 051802(R) (2001); E. Peik, J. Abel, Th. Becker, J. von Zanthier, and H. Walther, ibid. 60, 439 (1999).

[14] S. M. Barnett and S. Stenholm, J. Mod. Opt. 47, 2869 (2000). See also Phys. Rev. A 64, 033808 (2001), and references therein.

[15] H. J. Briegel and B.-G. Englert, Phys. Rev. A 47, 3311 (1993). For a review, see B.-G. Englert, and G. Morigi, in Coherent Evolution in Noisy Environments, Lecture Notes in Physics, Vol. 611, edited by A. Buchleitner, K. Hornberger (SpringerVerlag, Berlin, 2002), and references therein.
[16] M. Jakob and S. Stenholm, Phys. Rev. A 67, 032111 (2003).

[17] B. R. Mollow, Phys. Rev. 188, 1969 (1969).

[18] C. Cohen-Tannoudji, J. Dupont-Roc, and G. Grynberg, AtomPhoton Interactions (Wiley, Toronto, 1992).

[19] S. Stenholm, Rev. Mod. Phys. 58, 699 (1986).

[20] J. Eschner, G. Morigi, F. Schmidt-Kaler, and R. Blatt, J. Opt. Soc. Am. B 20, 1003 (2003).

[21] H. J. Carmichael, An Open Systems Approach to Quantum Optics (Springer-Verlag, Berlin, 1993).

[22] J. Javanainen, M. Lindberg, and S. Stenholm, J. Opt. Soc. Am. B 1, 111 (1984); M. Lindberg and S. Stenholm, J. Phys. B 17, 3375 (1985). 